\title{
Injection Moulded Microneedle Sensor for Real-Time Wireless pH Monitoring
}

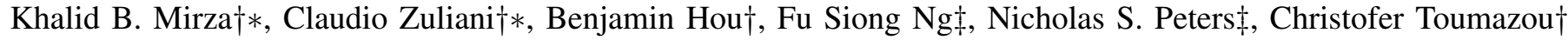 \\ $\dagger$ Department of Electrical and Electronic Engineering, Imperial College London, SW7 2BT, UK. \\ $\ddagger$ National Heart and Lung Institute, Imperial College London, W12 0NN, UK. \\ *First Authors, Email: k.mirza@imperial.ac.uk
}

\begin{abstract}
This paper describes the development of an array of individually addressable pH sensitive microneedles using injection moulding and their integration within a portable device for real-time wireless recording of $\mathrm{pH}$ distributions in biological samples. The fabricated microneedles are subjected to gold patterning followed by electrodeposition of iridium oxide to sensitize them to 0.07 units of $\mathrm{pH}$ change. Miniaturised electronics suitable for the sensors readout, analog-to-digital conversion and wireless transmission of the potentiometric data are embodied within the device, enabling it to measure real-time pH of soft biological samples such as muscles. In this paper, real-time recording of the cardiac $\mathrm{pH}$ distribution, during ischemia followed by reperfusion cycles in cardiac muscles of male Wistar rats has been demonstrated by using the microneedle array.
\end{abstract}

\section{INTRODUCTION}

The application of microneedle arrays in biomedical science ranges from drug delivery to detection of biomarkers [1]. In terms of microneedles fabrication, injection moulding emerged as an alternative process to silicon microfabrication offering advantages towards cost and robustness [2]. In complex systems such as muscles, organs and applications where food chemical heterogeneities have to be measured, in this regard, the microneedles can serve as minimally invasive tools to achieve high-density chemical mapping.

Iridium oxide ( $\mathrm{IrOx}$ ) emerged as a possible candidate for overcoming the major limitations of the glass electrode as $\mathrm{IrOx}$ microneedles enable ease of miniaturization and development of sensor format [3]. IrOx also exhibits a superNernstian response of $60-80 \mathrm{mV} / \mathrm{pH}$-unit across a wide $\mathrm{pH}$ range, rapid response times and good chemical selectivity [3]. Further discussions about the equilibrium interfacial reactions that account for this behaviour can be found elsewhere [4].

In this paper, injection moulding has been used as a fabrication procedure to develop microneedles on a polymer substrate. The microneedles were sensitized to detect $\mathrm{pH}$ change. It also discusses interface electronics to enable wireless transfer of $\mathrm{pH}$ data from the microneedles. The functionality of the device has been demonstrated in the context of muscular metabolism under conditions of lactic acidosis.

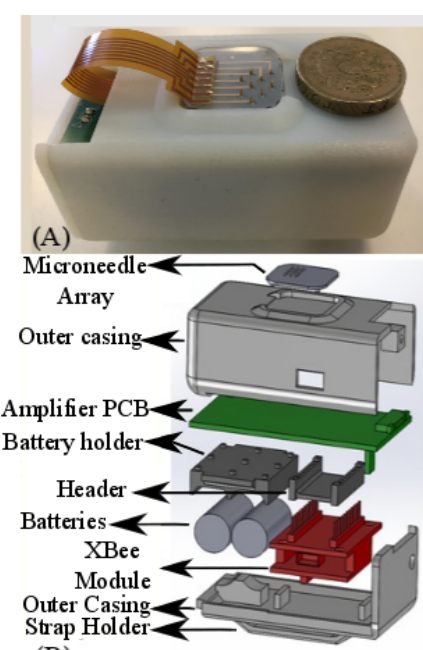

(B)

Fig. 1. (A) Photograph of the device in comparison to a $£ 1$ coin. (B) Internal stacking of different parts within the enclosure.

\section{SySTEM DESCRIPTION}

The microneedles aimed at recording $\mathrm{pH}$ are developed using injection moulding technique. This helps in using standard polymer material and leads to a reduction of overall fabrication cost. The microneedles, after functionalisation are interfaced to board level electronics to enable real time data acquistion and wireless transmission. The materials needed and the methods involved in developing the $\mathrm{pH}$ sensitive microneedles are described, followed by a detailed description of the system electronics.

\section{A. Microneedle Fabrication}

A 3D model of the microneedle arrays was prepared using Solidworks. Polyethylene terephthalate (PET) microneedle arrays were fabricated using injection moulding. The distances (center-to-center) among the microneedles is $2.2 \mathrm{~mm}$ in the array and are arranged in a triangular pattern and . As specified in [5], the activation procedure involved covering PET microneedles with laser cut Mylar sheet. At this stage, only the microneedles and contact tracks were exposed and sputtered with gold. A flexible customised cable was attached to the conductive contact tracks of the microneedles through conductive epoxy. After curing, IrOx was deposited 


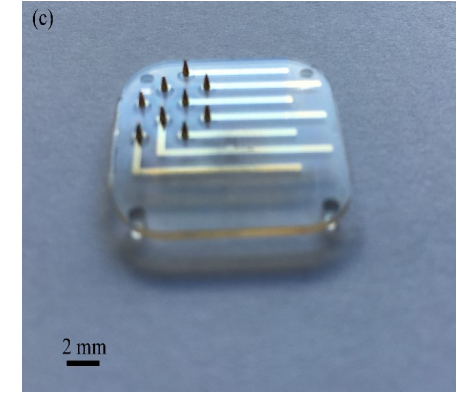

Fig. 2. Photograph of the microneedle array. The radius and height of the needles are $0.8 \mathrm{~mm}$ and $1.6 \mathrm{~mm}$ respectively, tip diameter is $50-80 \mu \mathrm{m}$ and the inter-needle pitch (center-to-center) is $2.2 \mathrm{~mm}$

electrochemically using a modified version of the protocol reported in [5].

The enclosure for the device was modelled using SolidWorks and printed using the Objet Connex 260 printer using the vero white resin. Although this spatial arrangement was chosen for convenience in the manufacture process rather than physiological considerations, it allows mapping $\mathrm{pH}$ heterogeneities across a relatively large area.

\section{B. Data Acquisition}

A customized flexible cable which ensured electrical contact between the needles and the electronics was manufactured by Digi International. A PCB with front-end amplifier, LTC6079, was used to interface with the microneedles. XBee communication protocol was implemented for communication with two xbee PRO S2 zigbee modules. These modules were used as transmitter ( $\mathrm{Tx})$ and receiver $(\mathrm{Rx})$ for the wireless acquisition of the potentiometric data.

LTC6079 was chosen since, it is a multichannel amplifier(4 channels in one chip), it has high input impedance, $10{ }^{15} \Omega$, and very low input bias current, ca. 200 fA. This reduces degradation of sensors and also reduces sensor drift. The inputs of LTC6079 were protected using guard rings on PCB to minimise current leakage and hence, prevent measurement errors. As the rate of $\mathrm{pH}$ signal change is very low ,the output of LTC6079 was low pass filtered (2 Hz cut-off) using a first order low pass filter implemented to avoid aliasing and level-shifted to interface with the on-chip analogue to digital converter (ADCs) of SparkFun XBee Explorer board.

The amplifier was powered up in dual supply configuration (+/-3V) using two rechargeable batteries. An mbed microcontroller board from ARM enabled the connection between the Rx xbee and a PC terminal. A SparkFun XBee Explorer board allowed the connection between the Tx xbee and the amplifier PCB. The Tx xbee seats onto the XBee Explorer board which provides breakout headers to connect the custom PCB to the xbees I/O pins and to a USB connector. Six connections were made between the custom PCB board and the xbee: $+5 \mathrm{~V}$ and ground (GND) and 4 voltage signals (from the PCB to the xbee pins ranging from 17 to 20). The Rx xbee and mbed seat onto the mbed application board which provides the necessary connections between the mbed and the xbee and the diagnostic modules, e.g., LCD screen. The mbed application board is connected to a PC terminal via USB for power and serial data communication.

The Rx and the Tx xbee modules were configured as coordinator and as router, respectively, using Digis XCTU program. Both xbees are set with an identical PAN ID (Personal Area Network Identification) thus allowing them to communicate with each other. Pins AD0 to AD3 on the Tx xbee were set to analogue input with 10 bit sampling and a sampling rate of $5 \mathrm{~Hz}$. This helps to avoid $50 \mathrm{~Hz}$ interference. The ADC sampling is performed simultaneously to sample acquisition and it is then transmitted to the Rx xbee ( 4 samples in 1 data packet) which stores the data in its internal buffer. The program on the mbed fetches a packet from the Rx xbee, dissects the latter and sends it to the computer terminal via USB and also displays it on the LCD screen. A python script is executed on the PC terminal to detrend the incoming data removing electrode drift, log the incoming data to a CSV file and to provide a real-time plot.

The zigbee platform was chosen due to the low-power and the transmission data rate required, i.e., 4 channels at 10 bits per channel at $5 \mathrm{~Hz}$ yield a data rate of 200 bits per second which is within the Zigbee specifications ( $250 \mathrm{kbit} / \mathrm{s}$ as maximum transmission data rate). In terms of power consumption xbee has a peak Tx current of $295 \mathrm{~mA}$ and a powerdown current of the order of $10 \mu \mathrm{A}$. The unit is powered down when not transmitting. The maximum transmission rate for the application is 50 bits per second. If needed, under continuous operation and maximum transmission rate, the capacity of two rechargeable batteries (100 mAh each) allows a maximum 40 minutes of continuous transmission.

After testing the electronics and demonstrating the feasibility of the array of the individually addressable IrOx coated microneedles for monitoring $\mathrm{pH}$ in a biological sample, the work focussed on the integration of the these components in order to deliver a functional device. The 3D-printed case allows securing the microneedle array on the outer surface and it also allows the housing of the analog electronics, wireless module and batteries. Fig. 1 reports the 3D model highlighting the integration of the components into the final prototype. As shown in Fig. 2, although nine microneedles were realised, eight microneedles could be interfaced with connections, leading to an eight channel electronic system. Fig. 3 shows the system architecture of the wireless $\mathrm{pH}$ recording system.

\section{RESUlTS}

The device was tested on the bench to determine $\mathrm{pH}$ sensitivity of the microneedles and also device functionality using $\mathrm{pH}$ buffers of known $\mathrm{pH}$ values. The device was also tested in vivo to record $\mathrm{pH}$ in explanted heart of male Wistar rats in which ischaemia was induced to trigger anaerobic glycolysis leading to $\mathrm{pH}$ change, followed by reperfusion. All studies were approved by the institutional animal care and use committee of Imperial College London. 


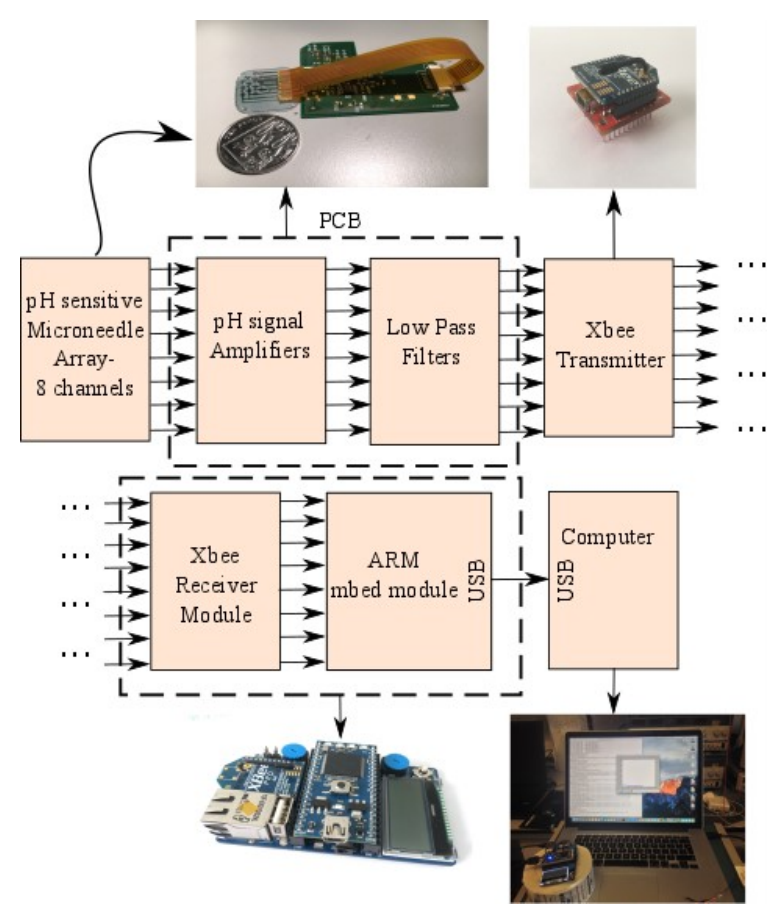

Fig. 3. A system level diagram showing different functional blocks in the wireless $\mathrm{pH}$ recording system.

\section{A. Bench Measurements}

The $\mathrm{pH}$ of all the solutions was measured using a $\mathrm{pH}$ meter from Hanna (Sigma-Aldrich, UK). The potentiometric traces of the $\mathrm{pH}$ sensors were recorded under quiescent conditions using the EMF-16 high-input impedance voltmeter (Lawson, USA) and a double junction $\mathrm{Ag} / \mathrm{AgCl}$ reference electrode (Sigma-Aldrich, UK) filled with an inner solution of 4.0 $\mathrm{M} \mathrm{KCl}$ and an outer solution of $1.0 \mathrm{M} \mathrm{KNO3.} \mathrm{It} \mathrm{should}$ be noted that employing a double junction $\mathrm{Ag} / \mathrm{AgCl}$ and using the same buffer for bathing and perfusing the heart minimizes the contribution of the junction potentials across the phase boundaries. In order to determine the sensitivity of the microneedles, calibration was performed using buffers of known $\mathrm{pH}$, ranging from $\mathrm{pH} 4-\mathrm{pH}$ 8. The voltage output was noted and a linear estimation was performed to determine sensitivity according to the Nernst equation.

Fig. 4 compares the calibration curve as obtained from a microneedle of the array using the bench-top high-input impedance voltmeter and the in-house electronics. It is apparent that the slope values of the two calibration trends are identical, i.e., $63 \mathrm{mV} / \mathrm{pH}$ unit, however, the offset values differ significantly. This gap is probably due to differences in input stages of the LTC6079 and the bench-top voltmeter. It is perhaps important to note that a gain of 3 for the frontend amplifier was found optimal for this application. In fact, this gain in conjunction with the resolution of the ADC, i.e., $12.9 \mathrm{mV}$, allows a resolution of at least $0.07 \mathrm{pH}$ unit change since the sensitivity of electrodes is typically larger than $60 \mathrm{mV} / \mathrm{pH}$ unit. In addition, the above gain ensures that the largest signal is within the input range of the ADC $(3.3 \mathrm{~V})$ since the DC offset between the sensors and the reference electrode does not typically exceed $600 \mathrm{mV}$. The upper limit of measurable $\mathrm{pH}$ change detected by the system is determined by the DC offset and the voltage rail used as shown in Equation 1.

$$
\Delta V_{p H} \leq \frac{V_{\text {supply }}}{\text { Gain }}-\text { Offset }
$$

$\Delta V_{p H}$ is the voltage difference between $\mathrm{pH}$ electrode and reference electrode originating from change in $\mathrm{pH}$. $V_{\text {rail }}$ is the supply voltage which in this case is $3.3 \mathrm{~V}$. Considering the worst case DC offset of $600 \mathrm{mV}$ and amplifier gain of 3, $\Delta V_{p H}$ is calculated to be $500 \mathrm{mV}$. This translates to a $\mathrm{pH}$ change of $7.9 \mathrm{pH}$ units for an electrode of $63 \mathrm{mV} / \mathrm{pH}$ unit sensitivity. Hence, the system can detect changes ranging from 0.07-7.9 $\mathrm{pH}$ units.

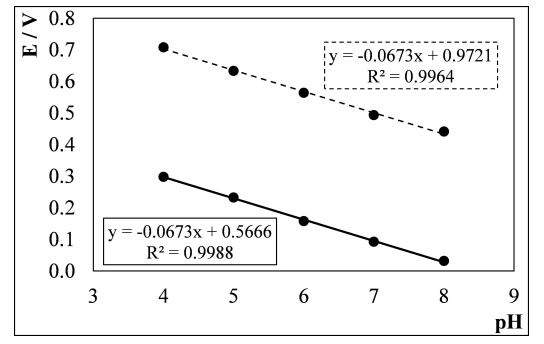

Fig. 4. Comparison of calibration trends obtained with one of the microneedles in the array using bench-top equipment (dotted) and the inhouse developed electronics (solid) consisting of the analogue and wireless module.

\section{B. In vivo Results}

The heart slices were imaged with a ZeissAxio Observer wide-open field microscope following histopathological treatment as specified in [5]. Electrocardiogram (ECG) was monitored throughout the ex-vivo experiment using LabChart and a bioamplifier (both AD Instruments, New Zealand). The experiment protocol followed was similar to the protocol described in [5]. A pneumatically-actuated inserter (Blackrock, USA) with a $1.0 \mathrm{~mm}$ piston, generally used as a means of precise insertion device form microelectrode arrays, was employed to insert the microneedles array into the heart.

1) Drift Analysis and Processing: The drift of the electrode was studied, post impalement, to enable on-going processing of data during the experiment. The drift was observed to be approximately linear, as shown in Fig. 5. Hence, on the receiving computer, the linear estimation was performed on 60 minutes of data. This linear estimation was then subtracted from the raw data to remove the effect of electrode drift. The heart beat artefact on the $\mathrm{pH}$ recording is not visible as the heartrate of rat is around 330-480 beats/minute or minimum 5 beats/second approximately. As the sampling rate of the system is $5 \mathrm{~Hz}$, the heart rate artefact is not pronounced on the $\mathrm{pH}$ recording.

2) Ischaemia experiments: Fig. 6 shows the real-time $\mathrm{pH}$ changes taking place across the whole heart during the ischemia/reperfusion cycles. In fact, the $\mathrm{pH}$ drops and reverts to the initial values as soon as the coronary perfusion is 


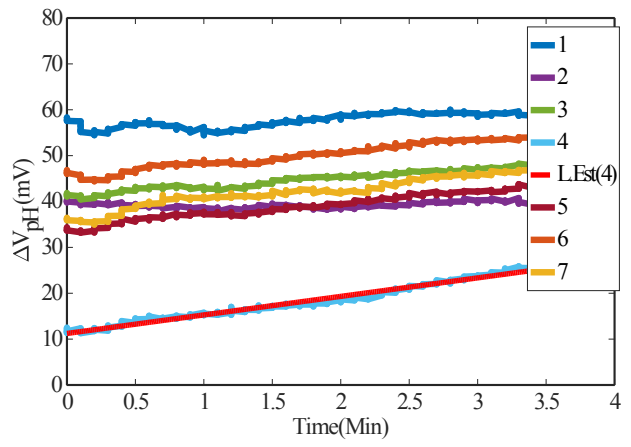

Fig. 5. Electrode drift observed in microneedles post-impalement with explanted heart present in the perfusate. The drift characteristics can be approximated to be linear (red), as shown for microneedle 4 (dotted).

stopped and as soon as the reperfusion of the ischemic myocardium is re-started, respectively. However, the magnitude and the dynamics of these variations depend on the individual sampling site.

Fig. 7 demonstrates that the electrocardiograms recorded before and after the array impalement were unchanged, i.e., no changes to the heart rate, QRS complex morphology or the ST segment/T wave. This result suggests that the impalement of the microneedles did not affect the electrophysiology of the heart.

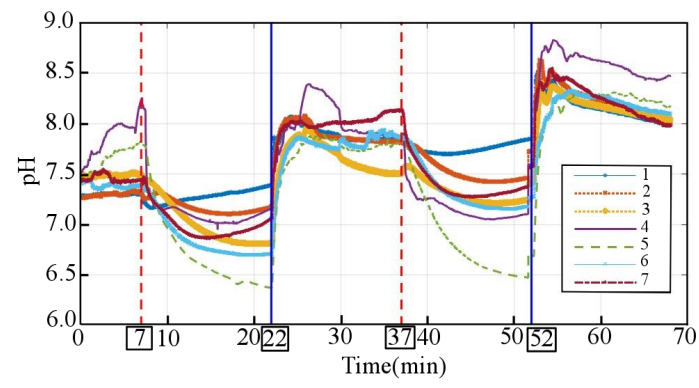

Fig. 6. $\mathrm{pH}$ changes obtained with the microneedles device, inserted into the perfused rat heart. Each waveform shows the $\mathrm{pH}$ as measured by the seven sensors. It also shows fall and recovery of $\mathrm{pH}$ values after ischaemia and reperfusion respectively. The heart underwent two cycles of global heart ischemia/reperfusion, i.e., ischemia occurred at $\mathrm{t}=7 \mathrm{~min}$ and $37 \mathrm{~min}$ while reperfusion was started at $\mathrm{t}=22 \mathrm{~min}$ and $52 \mathrm{~min}$.

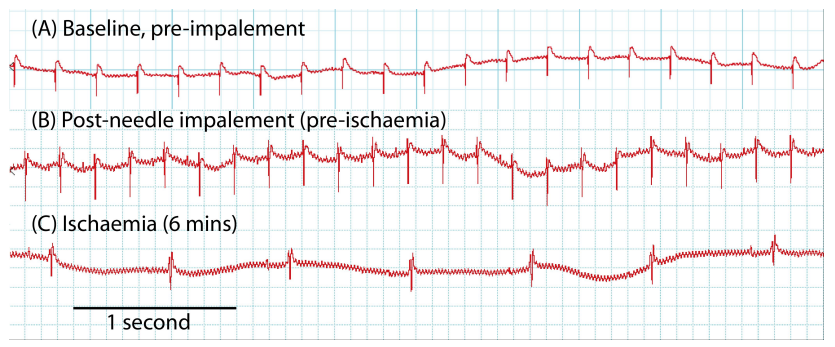

Fig. 7. Electrograms recorded: (A) before,(B) after impalement of microneedles array into the myocardium and (C) during global no-flow ischemia with the $\mathrm{pH}$ sensing device inserted in the heart.

\section{CONCLUSION}

The manuscript reported the preparation of a portable/wearable device for real-time monitoring of $\mathrm{pH}$ thanks to the integration of an array of individually addressable $\mathrm{pH}$ sensitive needles and wireless electronics. The use of injection moulded microneedles enables rapid prototyping at lower cost. The microneedles were inserted in an explanted perfused rat heart in order to demonstrate that the array could record the existence of a complex cardiac microenvironment as observed during the cycles of ischemia/reperfusion. Overall, this device affords a novel low-cost analytical tool which may find applications as portable instrument for measurement of $\mathrm{pH}$ in biological samples such as organs and food or as a wearable for instance to monitor the $\mathrm{pH}$ of the muscles during physical exercise. In fact, the prototype prepared here measures and wirelessly transmits the $\mathrm{pH}$ data in real-time averaging the values harvested from all the sensors, i.e., microneedles, inserted in the sample.

Future work aims at reducing the size of the device and to shift towards multichannel analogue front-end integrated circuit (IC) with on-chip ADCs and wireless telemetry. Further considerations towards an implanted, chronic solution includes possibility of auto-calibration of $\mathrm{pH}$ sensors. In a biological system, the $\mathrm{pH}$ of blood is tightly regulated, hence can be used as a standard to determine the sensor sensitivity. However, determining the linearity of the implanted sensor becomes a challenge as this requires multiple known $\mathrm{pH}$ standards.

\section{ACKNOWLEDGMENT}

This work was supported by the European Research Council (Synergy Grant No. 319818, i2MOVE). We would like to thank FILM facilities at Imperial College for their help, support and assistance. Fu Siong Ng was supported by an NIHR Clinical Lectureship (1716) and an Academy of Medical Sciences Starter Grant (AMS-SGCL8-Ng).

\section{REFERENCES}

[1] L. M. Strambini, A. Longo, A. Diligenti, and G. Barillaro, "A minimally invasive microchip for transdermal injection/sampling applications,' Lab on a Chip, vol. 12, no. 18, pp. 3370-3379, 2012.

[2] H. Zhou, G. Li, X. Sun, Z. Zhu, B. Xu, Q. Jin, J. Zhao, and Q.-S. Ren, "A new process for fabricating tip-shaped polymer microstructure array with patterned metallic coatings," Sensors and Actuators A: Physical, vol. 150, no. 2, pp. 296-301, 2009.

[3] S. R. Ng and D. O'Hare, "An iridium oxide microelectrode for monitoring acute local ph changes of endothelial cells," Analyst, vol. 140, no. 12, pp. 4224-4231, 2015.

[4] P. Steegstra and E. Ahlberg, "Influence of oxidation state on the ph dependence of hydrous iridium oxide films," Electrochimica Acta, vol. 76, pp. 26-33, 2012.

[5] C. Zuliani, F. S. Ng, A. Alenda, A. Eftekhar, N. S. Peters, and C. Toumazou, "An array of individually addressable micro-needles for mapping ph distributions," Analyst, 2016. 